\title{
Aproximación al valor social de un colegio rural agrupado: el caso del CRA "Sierra de Alcaraz"
}

\section{Virginia Barba-Sánchez, Beatriz Calderón Milán, María José Calderón Milán, Gabriel Sebastián Rivera}

RESUMEN: La medición del valor social, más allá del valor económico generado por las organizaciones, despierta un interés creciente en todo tipo de entidades y, en especial, en aquellas organizaciones en las que el valor social que generan es su principal razón de ser. Sin embargo, su supervivencia se vincula a cumplir una serie de ratios numéricos relacionados con el coste que suponen mantener en activo estas organizaciones, que nada tiene que ver con el valor social que generan, difícilmente medible en términos cuantitativos. Este es el caso de los Colegios Rurales Agrupados (CRA), cuya existencia, además de otros beneficios, fomenta el arraigo al territorio, previniendo lo que se ha dado en denominar la España despoblada. En este contexto, se sitúa el presente trabajo, cuya primera contribución es aproximarnos al valor social generado por el CRA Sierra de Alcaraz (Albacete). La metodología que se sigue es el estudio del caso y el proceso de análisis se sustenta en el método SPOLY, especialmente indicado para organizaciones de no mercado, como es el caso de las instituciones educativas públicas. Así, en el trabajo se identifican los grupos de interés del CRA Sierra de Alcaraz y, por medio de una serie de entrevistas a los mismos, se definen las variables de valor orientadas a indicadores, cuya cuantificación permite aproximarnos al valor social generado por el CRA Sierra de Alcaraz y realizar un análisis de su eficiencia económica en términos sociales. Como resultado del trabajo, se obtiene que el valor social integrado casi duplica el valor socio-económico directo generado por esta entidad.

PALABRAS CLAVE: Monetización del valor social, stakeholders, SPOLY, colegio rural agrupado.

CLAVES ECONLIT: I26, L32, M41, 033.

Cómo citar este artículo/How to cite this article: BARBA-SÁNCHEZ, V., CALDERÓN, B, CALDERÓN, M.J. \& SEBASTIÁN, G. (2021): "Aproximación al valor social de un colegio rural agrupado: el caso del CRA Sierra de Alcaraz", CIRIEC-España, Revista de Economía Pública, Social y Cooperativa, 101, 85-114. DOI: 10.7203/CIRIEC-E.101.18098.

Correspondencia: Virginia Barba-Sánchez, Universidad de Castilla-La Mancha, Departamento de Administración de Empresas, virginia.barba@uclm.es, ORCID: orcid.org/0000-0003-0149-0569; Beatriz Calderón Milán, Universidad de Castilla-La Mancha, Departamento de Economía Española e Internacional, Econometría e Historia e Instituciones Económicas, Beatriz.Calderon@uclm.es, ORCID: orcid.org/0000-0002-9738-7672; María José Calderón Milán, Universidad de Castilla-La Mancha, Departamento de Economía Española e Internacional, Econometría e Historia e Instituciones Económicas, MariaJose.Calderon@uclm.es, ORCID: orcid.org/00000001-8829-8750; Gabriel Sebastián Rivera, Universidad de Castilla-La Mancha, Instituto de Investigación en Informática de Albacete, Gabriel.Sebastian@uclm.es, ORCID: orcid.org/0000-0002-1156-8000. 


\section{Expanded abstract}

\section{Approach to the Social Value of a Rural Cluster School: the Case of RCS "Sierra de Alcaraz"}

\section{Context and Objective}

Rural Cluster Schools (RCS) play a very important role in the provision of an essential and fundamental service such as education (infant and primary levels) in sparsely populated rural areas (less than 2,000 inhabitants) with a scattered population. RCSs emerged in Spain in 1986, as a result of Royal Decree 2731/1986 (Ministry of Education and Science, 1986) to avoid the closure of rural schools in those municipalities which, due to their small population, did not reach the minimum number of pupils required to continue functioning. RCSs consist of the grouping of several rural schools into a single educational centre which operates and maintains the location of the rural schools that make it up (called sections) and which share resources to achieve a more efficient and functional operation. The RCSs thus contribute to rural development, to social and territorial cohesion and to the fight against the very serious problem of depopulation suffered by Spain's sparsely populated rural municipalities. If the school, the health centre and, in short, the most basic and essential services in rural areas are closed, the villages end up disappearing. Therefore, the role of the RCSs goes beyond the provision of the non-market service, i.e., the infant and primary education service to the population where they are located.

In this context, this article consists of the analysis of a case study, the RCS "Sierra de Alcaraz", which groups together schools located in five localities in the southwest of the province of Albacete (Castilla-La Mancha, Spain, all of which are municipalities with less than 500 inhabitants). The case study is relevant to the extent that Castilla-La Mancha is one of the Spanish regions with the lowest population density, the highest concentration of population in sparsely populated areas and the one that is suffering the most from the phenomenon of depopulation in rural areas.

As the contribution of Rural Grouped Schools goes beyond the provision of the education service in the rural area where it is located, the aim of this article is the quantification and monetization of the social value generated by the RCS "Sierra de Alcaraz" during the year 2019. To achieve this objective, the use of social accounting and, specifically, the San-Joseand Retolaza's Polyhedral Model or SPOLY Model, is proposed. 


\section{Design and Methodology}

The methodology employed is based on the use of social accounting applied to the RCS "Sierra de Alcaraz", a public educational centre that provides a non-market service. The aim of social accounting is to quantify and monetise the social value generated by an organization beyond its Gross Value Added (GVA).

The methodology used to measure and monetize the social value generated by the RCS "Sierra de Alcaraz" is the San-Jose and Retolaza's Polyhedral Model or SPOLY Model. This model is based on Freeman's Stakeholder Theory and the concept of shared social value. (1984), as well as in Porter and Kramer's concept of shared social value. (2011). This makes it possible to establish a set of specific indicators to quantify the social value perceived by an organization's stakeholders.

Following the Polyhedral Model, the process and methodology for measuring and monetizing the integrated social value of the RCS "Sierra de Alcaraz" was as follows: Firstly, the GVA generated or direct socio-economic value was quantified by using data from the financial accounts of the entity under study; secondly, the knock-on effects that the entity under study induces through its purchases from suppliers, also known as mobilized value or indirect socio-economic value, were quantified. The sum of these two elements makes up the social market value. Thirdly, the specific social value has been quantified. To do this, a map of the organization's stakeholders was drawn up and, through a series of in-depth interviews, the variables of value that the organization has on all its stakeholders were identified. These value variables are translated into indicators and proxies whose monetary quantification makes it possible to approximate the specific social value. Finally, the sum of the specific social value and the social market value will make up the integrated social value (ISV). The authors of the Polyhedral Model also add the emotional value but, due to its high subjectivity, it has not been possible to approximate it in this article.

\section{Results and Conclusions}

This article quantifies and monetizes the Integrated Social Value (ISV) of the Rural Grouped School "Sierra de Alcaraz" in 2019, which amounted to a total of €1,624,487. 52.8\% corresponded to the market social value (direct and indirect socio-economic value) and the remaining $47.2 \%$ to the specific social value. This shows that for every euro of added value generated by the RCS Sierra de Alcaraz, 0.91 euro of specific social value is generated for all its stakeholders. Moreover, for every euro that the Public Administrations allocate to finance this centre, the RCS Sierra de Alcaraz returns a value to society of at least $€ 1.96$. In this sense, those responsible for educational and territorial management policies should consider the results obtained by the RCSs, in terms of social profitability and not only cost, when deciding on their creation, maintenance or closure in a given region. 


\section{Original Value and Limitations}

The main contribution and originality of this article is to contribute to the visibility of RCSs. In Spain there is an almost total absence of statistical information and theoretical and/or empirical work on RCSs. Another innovation is the application of the Polyhedral Model for the quantification of the integrated social value to a Rural Cluster School, since until now this methodology had been applied to different types of organizations but never to a RCS.

One of the main contributions of this article and of all those that attempt to approximate the social value of organizations is to highlight the need to measure the value generated by an entity, which goes far beyond the value reflected in their Gross Value Added figures, and to quantify and monetize it.

In this sense, progress needs to be made in the development of methodologies that account for the social value of all types of organizations in order to quantify all those elements of value that GVA does not measure. The Polyhedral Model is one of these methodologies and its merits lie in the mere fact of trying to measure social value, because everything that is not measured is invisible. However, both the Polyhedral Model and the rest of the Social Accounting methodologies must advance in the systematization and standardization of indicators, not only to reduce the cost of their compilation at a microeconomic level, which makes their extrapolation to a macroeconomic level difficult, but also so that, as it occurs with the financial accounting of organizations and the National Accounts of countries, we all can measure the same thing in the same way and the magnitudes that are extracted from this accounting process are analogous and comparable.

Keywords: Monetising social value; stakeholders; SPOLY; Rural Cluster School.

Financiación. Este trabajo ha sido parcialmente financiado por la Junta de Comunidades de Castilla-La Mancha (España) y cofinanciado por el Fondo Europeo de Desarrollo Regional, con motivo del proyecto de investigación "Avances Tecnológicos Aplicados a dar soporte y mejorar la actividad docente en Colegios Rurales Agregados" con acrónimo "TecnoCRA" (ref: SBPLY/17/180501/000495). 


\section{Introducción'}

La medición del valor social, más allá del valor puramente económico, es especialmente importante para aquellas organizaciones y entidades que producen bienes y servicios gratuitos o cuasi gratuitos ${ }^{2}$, como las Administraciones Públicas y las instituciones privadas sin fines de lucro al servicio de los hogares. Este tipo de entidades, al financiarse principalmente vía transferencias corrientes (impuestos, donaciones o transferencias de otras entidades, entre otras) y no mediante un precio representativo de mercado, la sociedad, en general, y los consumidores de sus bienes y servicios, en particular, no perciben ni valoran el "precio de lo gratuito" (Vidal Martínez, 2014). Además, el valor generado por este tipo de entidades va más allá de la ya de por sí difícil cuantificación de los bienes y servicios no de mercado tangibles que producen, sino que la mayor parte del valor generado por este tipo de entidades deriva de la producción de intangibles (por ejemplo, cohesión social, arraigo de la población al territorio, sentido de pertenencia al grupo o enseñanza en valores) que, en ocasiones, supera al anterior y es la verdadera razón de ser de este tipo de entidades (Canals, 2014).

Sin embargo, la supervivencia de este tipo de entidades se vincula a cumplir una serie de ratios numéricos relacionados con el coste que suponen mantener en activo estas organizaciones, que nada tienen que ver con el valor social que generan, difícilmente medible en términos cuantitativos. En este contexto, se encuadra el presente artículo, en el de la cuantificación del valor social de un colegio rural agrupado situado en la provincia de Albacete, - el Colegio Rural Agrupado Sierra de Alcaraz-.

Para conseguir este objetivo, el trabajo se estructura de la siguiente manera: en primer lugar, se analiza el contexto de los colegios rurales agrupados, para tratar de destacar aquellos intangibles que generan valor social por este tipo de entidades; en segundo lugar, se analiza el marco teórico y metodológico. Como planteamiento teórico de medición de ese valor social se utiliza la Teoría de los Stakeholders de Freeman (1984) y el concepto de valor social compartido de Porter y Kramer (2011) y como marco metodológico de análisis el Modelo Poliédrico de San-Jose y Retolaza (2016) en el ámbito del estudio del caso. En tercer lugar, se realiza una descripción del caso de estudio CRA Sierra de Alcaraz. Y, en cuarto lugar, se identifican los grupos de interés o stakeholders del CRA Sierra de Alcaraz y, por medio de una serie de entrevistas a los principales stakeholders implicados, se identifican las variables de valor orientadas a indicadores, cuya cuantificación permitirá aproximarnos al valor económico y social generado por el CRA Sierra de Alcaraz. Finalmente, se recopilan las principales conclusiones y trabajos futuros.

1. Agradecemos la colaboración de todos los profesores, estudiantes, familiares, alcaldes y demás participantes, y muy en especial el apoyo incansable del equipo directivo del CRA Sierra de Alcaraz, sin el cual no hubiera sido posible este estudio. Además, agradecemos la labor previa de GEAccounting en el desarrollo, mejora y transmisión del método SPOLY para monetizar el valor social.

2. Son los denominados en la Contabilidad Nacional, bienes y servicios no de mercado o no destinados a la venta, cuyos productores no cubren al menos el 50\% de los costes de producción con el precio de venta del producto en el mercado. Mientras que los bienes y servicios de mercado o destinados a la venta serían aquellos cuyos productores cubren más del 50\% de los costes de producción con el precio de venta del producto en el mercado. A este precio representativo que cubre la mayor parte de los costes de producción se le denomina precio de mercado. 


\section{Contexto y conceptualización de los Colegios Rurales Agrupados y elementos de valor que generan}

El origen de los CRA en España se sitúa en el Real Decreto 2731/1986 (Ministerio de Educación y Ciencia, 1986) cuyo objetivo principal fue dar vía legal a organizaciones educativas diferentes y a la vez beneficiosas para la educación en el ámbito rural. Este Real Decreto permite, por primera vez, la posibilidad de constituir un CRA como fórmula organizativa más eficiente y funcional de la escuela rural y dotarla así de unas características propias, impregnándola de significado e importancia (Consejo Escolar del Estado, 2019; Lacort Navarro, 2014; Domingo Cebrián, 2013; Ponce de León Elizondo, Bravo Sáinz, \& Torroba Santa María, 2000) a la vez que trata de garantizar la provisión de servicios públicos esenciales (como la educación) suficientes y de calidad en el medio rural (Ley 45/2007, de 13 de diciembre, para el Desarrollo sostenible en el medio rural (LDRS); Programa de Desarrollo Rural Sostenible (2010-2014); (Domínguez Álvarez, 2020).

Un CRA es, a efectos de la Administración, un único centro educativo. Está conformado por un conjunto de colegios rurales próximos, denominados secciones, que están unidos para compartir recursos (profesorado especialista, materiales y proyectos, entre otros) y una única dirección que gestiona el centro y que se encuentra, normalmente, en la localidad de mayor importancia de la comarca o zona, que es la sede administrativa, conocida como cabecera (Lacort Navarro, 2014; Domingo Cebrián, 2013; Ponce de León Elizondo, Bravo Sáinz, \& Torroba Santa María, 2000). En Andalucía y Cataluña a este tipo de centros se les denomina, Colegio Público Rural (CPR) y Zonas Escolares Rurales (ZER), respectivamente.

Dentro de la zona de influencia de un CRA puede haber varias localidades con una única o varias unidades en las que pueden encontrarse alumnos de educación infantil y primaria en una única aula o, en otros casos, el número de alumnos es suficiente para realizar diversas agrupaciones. Estas agrupaciones generan aulas heterogéneas con unas necesidades y dinámicas muy diferentes de las de los centros educativos urbanos (Lacort Navarro, 2014; Ponce de León Elizondo, Bravo Sáinz, \& Torroba Santa María, 2000).

En la actualidad, los CRA son una fórmula organizativa educativa ya consolidada (Consejo Escolar del Estado, 2019), para la atención del alumnado de Educación Infantil y Educación Primaria, especialmente adecuada para la población que vive en localidades con población dispersa, de pequeño tamaño (municipios de menos de 2.000 habitantes) y en diseminado. El número de CRA en España, aunque con pequeños altibajos durante los últimos años, no ha dejado de aumentar, pasando de los 346 existentes a mediados de la década de 1990 (curso 1995-96) (Domingo Cebrián, 2013) a los 714 actuales (curso 2017-18) (Consejo Escolar del Estado, 2019), alcanzándose el máximo histórico durante el curso 2014-2015 con un total de 928 (Consejo Escolar del Estado, 2016). En cuanto a la evolución del número de alumnos en este tipo de centros y su peso relativo sobre el total de alumnos de estas enseñanzas, ha segui- 
do una evolución similar al del número de CRA, en concreto, aumentó hasta alcanzar el máximo histórico durante el curso 2014-2015 (78.721 alumnos; 1,8\% del total, respectivamente) y desde entonces ha ido disminuyendo hasta los últimos datos disponibles del curso 2017-2018 (71.727 alumnos; 0,9\% del total, respectivamente). En Castilla-La Mancha, aunque el número de alumnos en CRA no ha dejado de decrecer en la última década (alrededor de 10.000 curso 2011-12 a los poco más de 8.000 actuales, curso 2017-18), se mantiene la participación de este tipo de alumnado sobre el total de alumnos de estas enseñanzas (alrededor 11,4\%), siendo muy superior dicha participación a la media nacional $(0,9 \%$, ya se ha dicho) y aumenta el número de CRA (de 74 a 77).

Los CRA en España no pueden entenderse sin hacer referencia al fenómeno de la despoblación de las zonas rurales, lo que hoy en día se conoce con los términos de España vaciada o despoblada (Domínguez Álvarez, 2020; Escribano Pizarro, 2012; Collantes, Pinilla, Sáez, \& Silvestre, 2010; Escalona Orcao \& Díez Cornago, 2005). Según el Banco Mundial y los Censos de Población del INE, desde la década de 1960 a la actualidad, disminuye la población rural ${ }^{3}$ en España, tanto en términos absolutos como relativos (13,2 millones y 43,4\% población total en 1960; 9,2 millones y 19,7\% población total en 2018), esta tendencia solo se revierte durante el período 1992-2008 gracias al fenómeno de la inmigración (Collantes, Pinilla, Sáez, \& Silvestre, 2010). Este fenómeno de la despoblación rural tiene mucho que ver con las más bajas oportunidades de producción y empleo y de desarrollo del proyecto vital en estas zonas y, por ende, las más bajas tasas de crecimiento económico con respecto a las zonas urbanas y con una menor provisión de servicios públicos esenciales, como la sanidad, la educación, los servicios sociales, que aumentan los costes económicos y temporales de acceso de los usuarios y de los profesionales que prestan estos servicios, lo que contribuye todavía más a acrecentar el problema de la despoblación de las zonas rurales.

En este sentido, algunos autores (Domínguez Álvarez, 2020; Escalona Orcao \& Díez Cornago, 2005; Boix, 2003) destacan la importancia de la provisión de servicios públicos esenciales en el medio rural, en general, y de los servicios sanitarios y educativos, en particular, como herramienta indispensable para paliar el fenómeno de la despoblación.

Por su parte, el Consejo Económico y Social (2018) señala que entre los muchos factores que determinan la calidad de vida en el medio rural, el funcionamiento de los servicios educativos y sanitarios es de vital importancia. Sin embargo, también destaca que la presencia de estos servicios públicos esenciales es una condición, necesaria pero no suficiente, para atraer población a un municipio rural, debido a que la población que decide instalarse en un municipio rural suele ser población adulta y joven con un alto grado de movilidad que, más que valorar la existencia de este tipo de servicios, valora su calidad y tener un acceso rápido y cómodo a los mismos. En contraposición, la presencia de estos servicios públicos esenciales sí que es clave y determinante para mantener la estabilidad demográfica y a la población ya existente, debido a las mayores limitaciones de movilidad de la población que reside en ellos y a que la ausencia

3. En los Censos de población se entiende población rural aquella que reside en municipios de menos de 2.000 habitantes. 
de estos servicios sí que puede ser un factor de expulsión de la población importante por los costes económicos y temporales de acceso a este tipo de servicios (Domínguez Álvarez, 2020).

De la misma forma, la Red de Áreas Escasamente Pobladas del Sur de Europa (SSPA) señala que frenar la despoblación del medio rural español pasa, en buena medida, porque el conjunto de la población, ya sea rural o urbana, perciba que la calidad de vida en el medio rural es al menos equiparable a la de los territorios más densamente poblados, y que vivir en un pueblo no implica sistemáticamente tener que renunciar a unos servicios educativos, sanitarios, 0 sociales suficientes y de calidad (SSPA, 2018; Domínguez Álvarez, 2020).

En la misma línea, la Ley 45/2007, de 13 de diciembre, para el Desarrollo sostenible en el medio rural, así como el Programa de Desarrollo Rural Sostenible (2010-2014) destacan la necesidad de mejorar el acceso de la población de las zonas rurales a unos servicios públicos y de calidad, ya que una de las debilidades de las zonas rurales es la escasez de servicios a la comunidad (sanidad, educación, cultura,...) por razones de economía de escala debidos a la baja densidad y al aislamiento (Domínguez Álvarez, 2020).

A raíz de todas estas argumentaciones teóricas, se pone de manifiesto el papel fundamental que desempeña la escuela rural, en general, y los CRA, en particular en las zonas rurales, en la medida que fomenta el arraigo al territorio, previniendo la despoblación ya que son un espacio estrechamente vinculado al territorio. Pero este no es el único elemento de valor de los CRA.

Numerosos trabajos revelan las potencialidades pedagógicas, relacionales y humanas de los CRA sobre los principales grupos de interés implicados en ellos (alumnos, profesores, padres, ayuntamientos, vecinos) que son los principales elementos de valor social de este tipo de centros (Lacort Navarro, 2014; Domingo Cebrián, 2013; Feu i Gelis, 2004; Boix, 2003; Ponce de León Elizondo, Bravo Sáinz, \& Torroba Santa María, 2000).

Boix (2003) resalta el papel de las ZER para conservar la propia identidad de las zonas rurales, en el sentido de que la escuela rural ofrece a sus alumnos estrategias y recursos que les ayudan a entender y respetar la cultura local, el entorno natural, la historia del pueblo, lengua, las costumbres locales, el oficio de sus habitantes al integrarlos en sus proyectos educativos (Boix, 2003, pág. 6). Como otras bonanzas añadidas de la escuela rural señala (Boix, 2003, pág. 7): promover las conexiones socio-educativas entre los miembros de comunidades cercanas; corresponsabilizar a los miembros de la comunidad de los problemas, dificultades y conflictos que se generan en el entorno escolar; hacer explícitas las correlaciones positivas entre identidad y sentido de la comunidad o la promoción cultural del territorio; dignificar la cultura del niño dentro de su propio entorno natural y social y crear espacios que permitan construir los fundamentos de una mejor calidad de vida rural.

En Feu i Gelis (2004), se destacan como principales ventajas y puntos fuertes de las escuelas rurales: su reducido tamaño, que hace que todos los alumnos se conozcan, se ayuden y colaboren entre sí; favorecer la experimentación educativa y de la pedagogía activa, ya que al ser para muchos profesores sus primeros destinos y descubrir que la realidad rural poco tiene que ver con la realidad para la que se les prepara durante su formación académica, se ven obligados a adaptarse, a experimentar, a crear e innovar para salir adelante; capacitar a los profesores para que sean profesores integrales y polivalentes, al ser responsables de más de un nivel 
educativo y hacer un poco de todo en el centro escolar; favorecer el trabajo colaborativo y en equipo por parte de los alumnos al ser grupos heterogéneos; favorecer la enseñanza personalizada e individualizada ya que aunque el profesorado se vea obligado a realizar un poco de todo en el centro y en el aula, a la vez la enseñanza es más personalizada e individualizada ya que la relación profesor-alumno es mucho más estrecha; ayudar a la vinculación del alumnado con el entorno, reforzar la cultura local, crear vida en el pueblo; propiciar la autonomía del alumnado, la adquisición del sentido del deber y el aprendizaje del control de los tiempos porque aunque la enseñanza es más personalizada, el tiempo del profesor con cada alumno es limitado y esto favorece la creación de este tipo de hábitos en el alumnado.

Finalmente, Domingo Cebrián (2013) analiza la situación de tres CRA de Aragón y aunque se muestra bastante crítico con el tema de la baja dotación de recursos materiales, personales e instalaciones de este tipo de centros, así como con la alta rotación y débil formación del profesorado, sí que, al igual que los trabajos anteriores, muestra muchas de las potencialidades y elementos de valor de este tipo de escuelas rurales sobre los distintos grupos de interés o stakeholders. Para los padres: el tener a sus hijos en casa o muy cerca; la atención más personalizada; el que sus hijos conozcan a los niños de otros pueblos y edades y que aprendan unos de otros; así como facilitar que la gente se quede a vivir en los pueblos. Para los alumnos, además: la relación de cercanía con el maestro, la relación entre alumnos es más intensa, ayuda a que sean felices en su entorno, en sus pueblos y como luego deben desplazarse para cursar la ESO a otras localidades son más abiertos al cambio. Para los profesores, aunque supone mucho más trabajo especialmente los primeros años de adaptación, es una lección de aprendizaje y de vida enorme; se sienten más valorados por los alumnos y los padres que en la escuela urbana, es un trabajo gratificante a pesar del sobreesfuerzo de trabajar con un grupo tan heterogéneo y les ayuda a ser profesores integrales y más polivalentes. Para el Ayuntamiento y el pueblo: mejora la calidad de la educación en el medio rural; permite que la dotación de materiales y la plantilla de personal sea mayor; y potencia que los alumnos de distintos pueblos se conozcan y se relacionen.

Por tanto, a la luz de todos estos trabajos, se pone de manifiesto que el valor de los CRA va más allá del valor económico vinculado al servicio educativo que prestan a sus alumnos, sino que generan un valor social de difícil cuantificación puesto que la mayoría de esos elementos de valor son activos intangibles: evitar el despoblamiento, crear sentido de pertenencia al grupo y a la comunidad en el que se insertan; las interrelaciones entre los habitantes de los distintos municipios, entre otros.

\section{Marco teórico y metodológico}

Este trabajo se enmarca en el contexto de la Contabilidad Social y, por tanto, en el de la cuantificación del valor social generado por una organización, que va más allá del valor añadido y del análisis coste-beneficio que arrojan los indicadores de su cuenta de pérdidas y ganancias. La medición del valor social es muy complicada, ya que supone la medición de un valor que va 
más allá del valor añadido (en términos de contabilidad nacional) producido por la entidad y que genera utilidades y valor a la sociedad y a los distintos agentes que se relacionan con esa organización (stakeholders).

La metodología que se sigue para medir ese valor social es el Modelo Poliédrico de San-Jose y Retolaza (Retolaza, San-Jose, \& Ruiz-Roqueñi, 2015; San-Jose \& Retolaza, Contabilidad Social orientada a los stakeholders. Perspectiva de la Administración Pública, 2016) que permite establecer un conjunto de indicadores específicos para cuantificar el valor social percibido por el conjunto de grupos de interés o stakeholders que se relacionan con la entidad objeto de estudio. Este modelo tiene su base en la Teoría de los stakeholders de Freeman (1984) y en el concepto de valor social compartido de Porter y Kramer (2011).

Desde la propuesta de la Teoría de los stakeholders por parte de Freeman en 1984 hasta la actualidad, esta ha ido progresivamente ganando relevancia y ha sido revisada en otros trabajos posteriores de Freeman con otros autores (Freeman, Wicks, \& Parmar, 2004; Agle, y otros, 2008; San-Jose, Retolaza, \& Freeman, 2017; Freeman, Retolaza, \& San-José, 2020). De hecho, en la actualidad, una parte mayoritaria de las mayores 500 empresas a nivel mundial apuestan por una orientación hacia el conjunto de los stakeholders (Jesus, 2020; WEF, 2020). La Teoría de los stakeholders, tiene su extensión en el concepto de valor social compartido de Porter y Kramer (2011), y considera que el resultado de una actividad sólo se transforma en valor cuando hay alguien de su mapa de stakeholders o grupos de interés que lo valora. Por tanto, el Modelo Poliédrico de San-Jose y Retolaza (2016) parte de la identificación de los grupos de interés de la organización, entendidos como todos aquellos que tienen expectativas e intereses legítimos en la misma para, a partir de la identificación de los intereses de cada grupo, elaborar un mapa general de intereses (variables de valor), a los que la organización intenta responder; y en cuya respuesta genera Valor Social (output generado por la cuantificación de esas variables de valor).

La utilidad del Modelo Poliédrico de San-Jose y Retolaza (2016) cuenta con suficientes evidencias empíricas en la cuantificación del valor social de entidades de todo tipo en nuestro país (Retolaza, Ruiz-Roqueñi, San-Jose, \& Barrutia, 2014; Retolaza, y otros, 2015; Guzmán Pérez, Mendoza Jiménez, Pérez Monteverde, \& Román Cervantes, 2018; Etxezarreta, Mendiguren, Díaz, \& Errasti, 2018; San-Jose, Retolaza, \& Bernal, 2019; Román Cervantes C. , Guzmán Pérez, Mendoza Jiménez, \& Pérez Monteverde, 2019; Ayuso, Sánchez, Retolaza, \& Figueras-Maz, 2020; Román Cervantes C. A., Guzmán Pérez, Mendoza Jiménez, \& Pérez Monteverde, 2020; Retolaza, Aguado y San-José, 2020). Si bien la mayoría de estos trabajos centran su objeto de estudio en entidades pertenecientes a la Economía Social y al Tercer Sector, la tendencia es que se haga extensiva a otro tipo de organizaciones, como las pertenecientes a las Administraciones Públicas y las empresas capitalistas. De hecho, aunque el trabajo pionero en este sentido fue la medición del valor social de la Fundación Lantegui Batuak, entidad centrada en la formación e integración laboral de personas con discapacidad, durante los últimos años, se ha cuantificado para otro tipo de organizaciones como las viviendas municipales y un hospital público de Bilbao; las cofradías de pescadores de Canarias, las Sociedades Agrarias de Transformación de Canarias; la Universidad Pública Pompeu Fabra; la Unión de Cooperativas Agrarias de Castilla-La Mancha y de Navarra, cooperativas sociales, entre otras. 
Según Guzmán et al. (2018) y Román Cervantes et al. (2020), el Modelo Poliédrico de San-Jose y Retolaza (2016) de Contabilidad Social se desarrolla sobre la base de un análisis coste-beneficio y de las cuatro hipótesis metodológicas siguientes: en primer lugar, un proceso de investigación participativo de action research (Lewin, 1946), se trata de una estrategia práctica que implica al conjunto de stakeholders en la resolución de problemas reales, mediante técnicas de investigación; en segundo lugar, una aproximación al concepto de valor social desde la Teoría de los stakeholders (Freeman, 1984), que permite detectar el valor social de una entidad sobre los grupos de interés que participan y se ven afectados por su actividad; en tercer lugar, la asunción de un paradigma fenomenológico, según el cual el valor no se define desde la propia organización, sino que los diferentes stakeholders identifican las dimensiones de valor que les genera la organización, mediante entrevistas en profundidad y cuestionarios. Estas dimensiones, identificadas por los perceptores de la actuación de la organización, se redefinen como variables de valor, vinculándolas a indicadores objetivables a través de proxys y; en cuarto lugar, la hipótesis de la lógica difusa, que consiste en establecer cuantificadores para las diversas variables de valor estimadas. Para cada variable de valor (conjunto difuso), se define una función de valor, que viene determinada por el conjunto de proxys identificados para cada variable de valor. El resultado es un área final que conforma el Modelo Poliédrico (o SPOLY) de San-Jose y Retolaza (2016) de análisis del valor social (Ilustración 1). Gráficamente se asemeja a una flor en el que el círculo interior representa el valor social que la organización genera y es reconocido como tal por los distintos grupos de interés. Por su parte, los pétalos reflejan el valor que es identificado de manera particular por un grupo de interés y que no confluye con el resto (Guzmán Pérez, Mendoza Jiménez, Pérez Monteverde, \& Román Cervantes, 2018; Román Cervantes C. A., Guzmán Pérez, Mendoza Jiménez, \& Pérez Monteverde, 2020).

Según el Modelo Poliédrico de San-Jose y Retolaza (2016), el Valor Social Integrado o Consolidado (blended value), que representa la consolidación del valor generado por la organización para el conjunto de stakeholders, está conformado por tres componentes: el valor socio-económico o valor social de mercado, el valor social específico o de no mercado y el valor emocional.

El valor social de mercado es el valor social que la organización genera con la realización de su actividad económica e incluye: por un lado, el valor añadido que, de forma directa, una organización genera y distribuye (valor añadido distribuido) y el valor generado y no distribuido (valor añadido retenido); y, por otro lado, el valor que, de forma indirecta, genera con las compras de insumos a proveedores (valor movilizado), ya que las compras a proveedores ejercen un efecto multiplicador en la comunidad y constituyen la base para la determinación del valor socio-económico indirecto (Guzmán Pérez, Mendoza Jiménez, Pérez Monteverde, \& Román Cervantes, 2018; Román Cervantes C. A., Guzmán Pérez, Mendoza Jiménez, \& Pérez Monteverde, 2020).

En lo que respecta al valor social específico, es el valor que la organización genera a cada grupo de interés. Para ello, es necesario construir el mapa de stakeholders de la entidad, realizar entrevistas a estos para identificar las variables de valor que, mediante la utilización de una serie de proxis, permitirán su medición. 


\section{Ilustración 1. Modelo Poliédrico de Análisis del Valor Social}

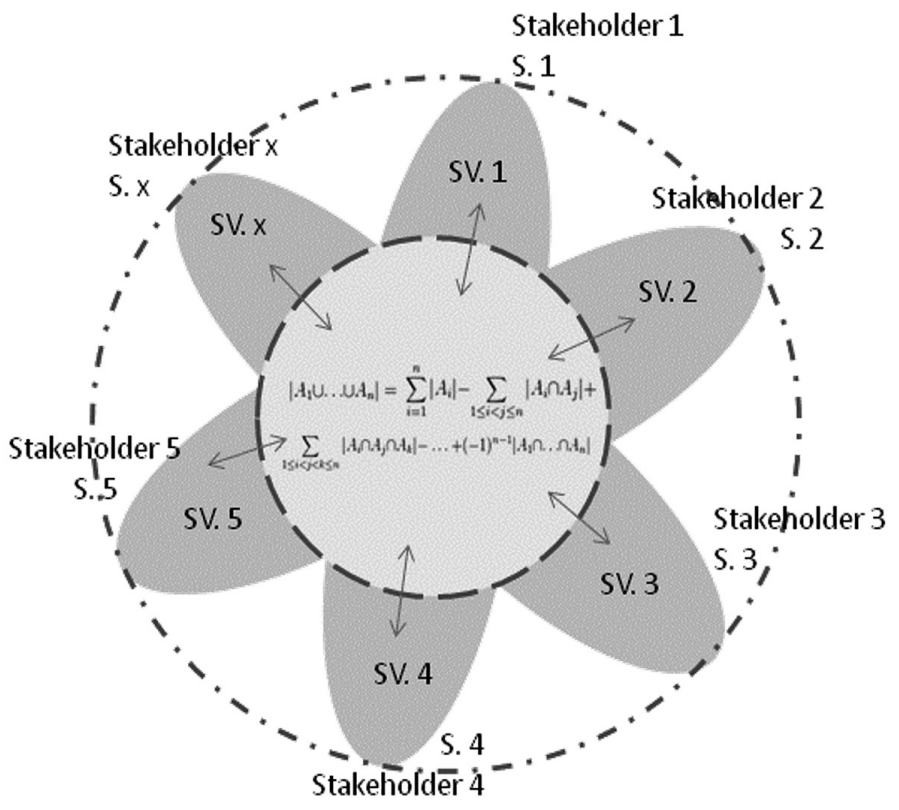

Fuente: Retolaza, San-Jose, \& Ruiz-Roqueñi (2016).

Y, finalmente, en lo que se refiere al valor emocional, recoge la satisfacción que la organización genera en sus grupos de interés.

\section{El caso de estudio: valor social del CRA "Sierra de Alcaraz"}

El CRA Sierra de Alcaraz es un centro público dependiente de la Junta de Comunidades de Castilla-La Mancha, situado al suroeste de la provincia de Albacete, donde se imparten las enseñanzas de 2o ciclo de Educación Infantil y de la etapa de Educación Primaria. Está integrado por 5 secciones (Ilustración 2) ubicadas en las localidades de: El Jardín, Peñascosa, Povedilla, Viveros y Robledo; siendo esta última la cabecera del centro, por ubicarse geográficamente centrada (la mayor distancia entre las distintas secciones y la cabecera es de $22 \mathrm{kms}$.). La población total de las localidades donde se ubican las cinco secciones oscila entre los 141 y los 430 habitantes (INE, 2020). La comarca en la que el centro está ubicado es la Sierra de Alcaraz, en la provincia de Albacete. 


\section{Ilustración 2. Localización geográfica de las 5 secciones del Colegio Rural Agrupado Sierra de Alcaraz y cabecera}

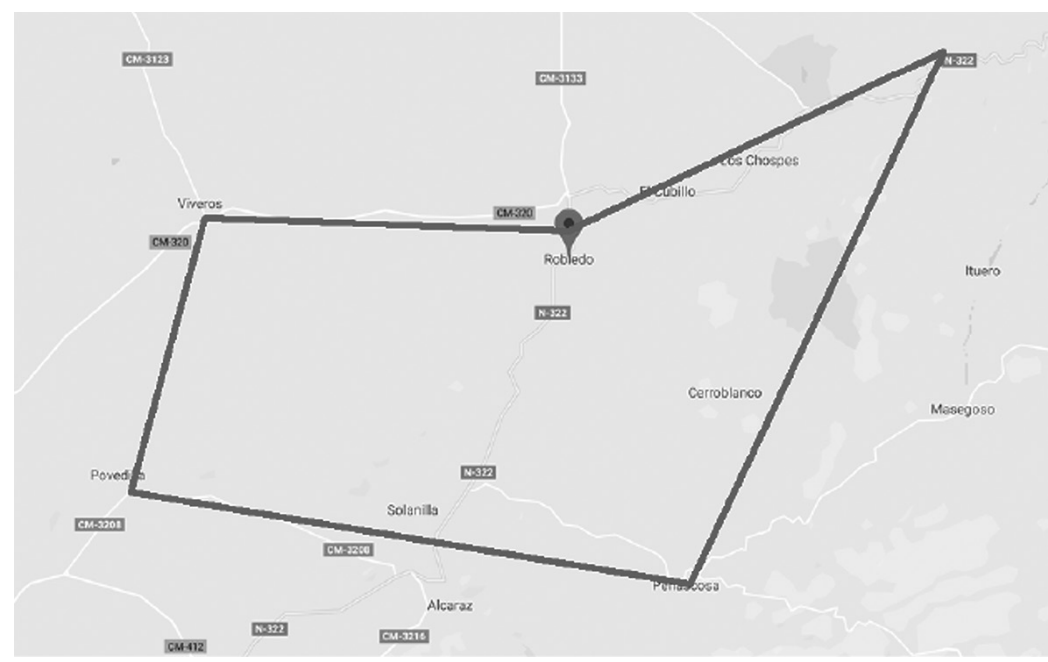

Fuente: Google maps (2020).

El CRA cuenta en la actualidad, con 10 unidades (3 de educación infantil y 7 de educación primaria) (Tabla 1), algo menos de 70 alumnos (12\% inmigrante y va en aumento) y 19 profesores, siendo parte de ellos itinerantes y a media jornada. Se cuenta además con dos rutas escolares con alumnos que se desplazan desde las localidades próximas de Zarzalejo, Los Chospes y las fincas de Viveros.

La base de la actividad económica de la comarca son las actividades agropecuarias, la caza y la explotación forestal. Aunque también existe alguna actividad industrial (entre las que destaca, una fábrica de aluminio, una planta embotelladora de agua y una fábrica de embutidos, además de una serie de pequeñas industrias ligadas directamente al sector agropecuario) y algunas actividades vinculadas con el turismo rural. La mayor parte de los alumnos egresados cursan la ESO en el Instituto de Alcaraz, aunque la tasa de abandono escolar temprano (antes de finalizar la ESO) se sitúa alrededor del $50 \%$. El nivel educativo de las familias es medio-bajo (la mayoría con estudios primarios y muy pocos con estudios medios y/o superiores). 
Tabla 1. Número de unidades del CRA Sierra de Alcaraz por secciones

\begin{tabular}{l|c|c} 
Sección & & Primaria \\
\hline Jardín & 1 & 2 \\
\hline Robledo & & 1 \\
\hline Peñascosa & 1 & 1 \\
\hline Povedilla & 1 & 2 \\
\hline Viveros &
\end{tabular}

Fuente: Elaboración propia.

\section{Resultados}

Para obtener el Valor Social Integrado generado por el CRA Sierra de Alcaraz y percibido por sus grupos de interés, es preciso determinar los tres elementos que lo conforman: el valor social de mercado, el valor social específico y el valor emocional. Dado el carácter experimental del proceso de cálculo del valor emocional propuesto por Ruiz-Roqueñi (2018) y con incipientes iniciativas por cuantificarlo por parte de Román et al. (2019), vamos a centrar nuestro análisis en los dos primeros. A continuación, se exponen los resultados para la entidad objeto de estudio en 2019.

\section{a. El Valor Social de Mercado}

El Valor Social de Mercado es el valor social que la organización genera con la realización de su actividad económica e incluye, por un lado, el valor añadido que, de forma directa, una organización genera (valor añadido distribuido y valor añadido retenido por la empresa) y, por otro lado, el valor que, de forma indirecta, genera con las compras de insumos a proveedores (valor movilizado).

\section{a1. El Valor Socio-económico Directo}

En la tabla 2 se recoge el Valor Socio-Económico Directo, calculado a partir de la metodología de Retolaza, San-José y Ruiz-Roqueñi (2016). Este valor asciende a un total de 847.608€, de los cuales un 92,66\% se distribuye de la siguiente forma: el 72,83\% entre los trabajadores en forma de sueldos y salarios, $19,47 \%$ entre las Administraciones Públicas en forma de cotizaciones a la Seguridad Social e IRPF y el 0,36\% en ayudas directas a las familias en forma de gratuidad de materiales curriculares y una beca de apoyo para un alumno con discapacidad. El 7,34\% restante es retenido por la Organización para aplicarlo en el siguiente ejercicio, dado el carácter no lucrativo de esta entidad. 


\section{Tabla 2. Valor Socio-Económico Directo del CRA Sierra de Alcaraz, 2019 (en $€$ )}

\begin{tabular}{l|c} 
Valor Socio-Económico Directo & $\mathbf{8 4 7 . 6 0 8} €$ \\
\hline Valor generado distribuido & $\mathbf{7 8 5 . 3 8 2 €}$ \\
\hline Salarios netos & $617.343 €$ \\
\hline Seguridad Social & $33.488 €$ \\
\hline IRPF & $131.545 €$ \\
\hline Ayudas directas a usuarios & $3.006 €$ \\
\hline Valor generado retenido & $62.226 €$ \\
\hline Retenido por la Organización & $62.226 €$ \\
\hline
\end{tabular}

Fuente: Elaboración propia.

\section{a2. El Valor Movilizado o valor socio-económico indirecto}

La actividad llevada a cabo por el CRA Sierra de Alcaraz conlleva una serie de compras a proveedores que contribuyen también, de forma indirecta, a la generación de valor y que, de no existir el CRA, no se llevarían a cabo. En este sentido, la metodología que estamos siguiendo en todo este proceso (Retolaza, San-Jose, \& Ruiz-Roqueñi, 2016), considera el valor generado por las compras a proveedores y que éstos distribuyen a su vez entre sus trabajadores, Administraciones Públicas (AA.PP.) y capital. Para obtener esta información, se han considerado todas las compras realizadas por el CRA Sierra de Alcaraz para el año 2019, analizando las características del proveedor (local o no) y el tipo de gasto (de explotación o de inversión).

La cartera de proveedores se ha buscado en la base de datos de la Central de Balances Ibéricos (SABI) para conocer su valor añadido, gastos de personal y otras variables de interés para el cálculo de los índices de repercusión que nos permiten realizar el reparto del valor generado a estos proveedores entre sus diferentes stakeholders. En SABI se ha conseguido localizar a los proveedores que suponen un $86 \%$ del total de compras realizadas por el CRA Sierra de Alcaraz durante el ejercicio 2019. Para calcular los índices de repercusión, se han considerado únicamente las cifras de los proveedores locales (situados en Albacete y su provincia) dado que un $70 \%$ del total de proveedores localizados en SABI cumplen esta característica y la estructura que nos proporcionan los índices de repercusión es más prudente que si consideramos el total de proveedores. El total de las compras a proveedores (locales y no locales) multiplicadas por los índices de repercusión, nos proporcionan el reparto del valor movilizado. Los resultados de la Tabla 3 nos indican el Valor Movilizado que el CRA Sierra de Alcaraz genera de forma indirecta en el ejercicio de su actividad económica, a través del gasto inducido a proveedores. Los proveedores han sido desagregados en dos grupos: los de explotación y los de inversión. El valor movilizado asciende a $9.540 €$, de los cuales el $69 \%$ es inducido sobre proveedores de 
explotación y el 31\% restante a proveedores de inversión. De este valor movilizado, el 60,1\% constituye un retorno a las Administraciones Públicas, en forma de cotizaciones a la Seguridad Social, IRPF, IVA y otros impuestos.

Tabla 3. Valor Movilizado por el CRA Sierra de Alcaraz, 2019 (en €)

\begin{tabular}{lr} 
Valor movilizado por proveedores & $9.540 €$ \\
\hline Valor movilizado por proveedores de explotación & $6.594 €$ \\
\hline Salarios netos & $2.071 €$ \\
\hline Seguridad Social & $1.711 €$ \\
\hline IRPF & $720 €$ \\
\hline IVA & $1.261 €$ \\
\hline Otros impuestos & $211 €$ \\
\hline Resultado del ejercicio & $620 €$ \\
\hline Valor movilizado por proveedores de inversión & $2.946 €$ \\
\hline Salarios netos & $977 €$ \\
\hline Seguridad Social & $844 €$ \\
\hline IRPF & $400 €$ \\
\hline IVA & $541 €$ \\
\hline Otros impuestos & $46 €$ \\
\hline Resultados del ejercicio & $138 €$ \\
\hline
\end{tabular}

Fuente: Elaboración propia.

\section{b. El Valor Social Específico}

El valor social específico o de no mercado del CRA Sierra de Alcaraz es el valor social que el colegio genera a sus distintos stakeholders. Dado que este colegio es público, la transmisión de valor de esta organización a su entorno se hace principalmente a través de mecanismos de no mercado, más allá de las transacciones monetarias (San-Jose, Retolaza, \& Bernal, 2019). Para su cálculo, el primer paso es identificar dichos grupos de interés tras varias reuniones con el equipo directivo del colegio, que se recogen de forma sintética en el mapa de stakeholders (Ilustración 3). 


\section{Ilustración 3. Mapa de Stakeholders del CRA Sierra de Alcaraz}

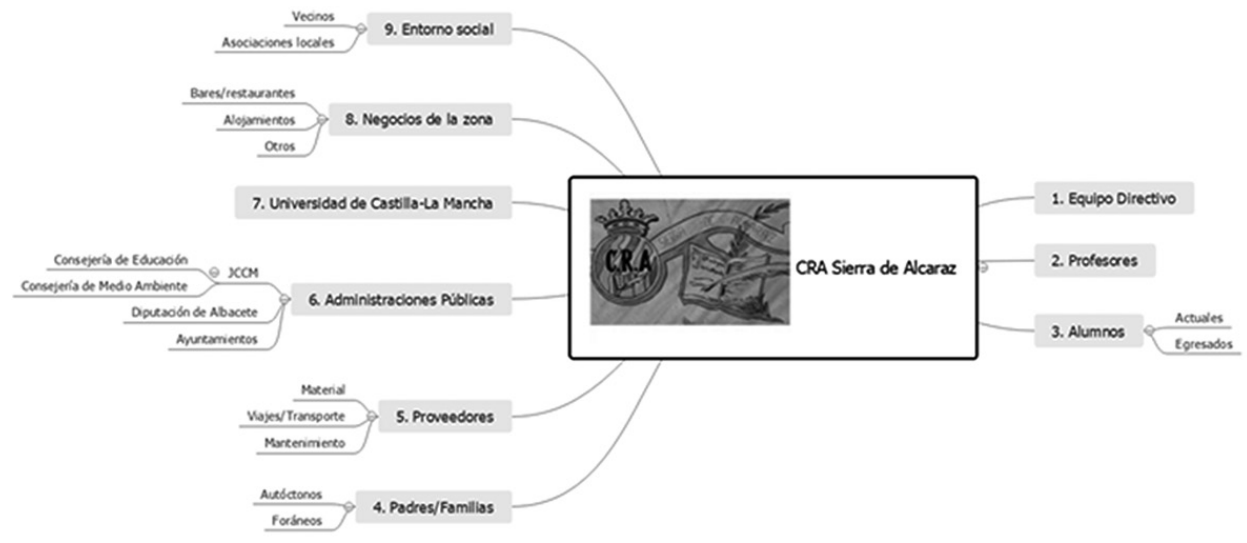

Fuente: Elaboración propia.

De forma detallada, los grupos de interés receptores del valor social de no mercado de este colegio son los siguientes:

- Alumnos. Este grupo es la esencia y la razón de ser del CRA. Son los niños/as que reciben el servicio educativo en los niveles de $2^{\circ}$ Ciclo de Infantil y de Educación Primaria y a los que se les estimulan ciertas habilidades y destrezas propios, no sólo del nivel educativo que reciben, sino de los valores inculcados por la escuela rural.

- Padres/familias. Grupo conformado por los familiares directos de los alumnos/as del CRA y que se benefician directamente de parte del valor social específico del mismo.

- $\quad$ Equipo directivo, profesores y demás personal del CRA, que realizan las labores de dirección y gestión y las propias del servicio educativo que prestan.

- Administraciones Públicas (AA.PP.). En el CRA Sierra de Alcaraz se ven implicados varios niveles de Administración: las Corporaciones Locales, como los Ayuntamientos de los municipios en los que se hallan las distintas secciones del CRA y la Diputación de Albacete, que se encargan principalmente de pagar los gastos corrientes y reparaciones del CRA y conceder ayudas y proyectos a distintas actividades que implican al CRA; la Junta de Comunidades de Castilla-La Mancha y las distintas Consejerías con competencias en materia de educación y medioambiente, que se encargan del pago de la nómina de los trabajadores y de cofinanciar proyectos y actividades del CRA; y la Administración Central que percibe la mayor parte de los ingresos inducidos por CRA vía pago de cotizaciones sociales e impuestos.

- La Universidad de Castilla-La Mancha que colabora en la realización de distintos proyectos de investigación sobre el CRA Sierra de Alcaraz. 
- Proveedores, que son aquellos productores que proveen de bienes y servicios corrientes y de inversión al CRA. En nuestro caso, distinguiremos los proveedores locales y los que operan a nivel nacional, puesto que entendemos que el CRA, a través de ellos, puede tener un mayor impacto en su entorno local.

- Negocios de la zona. Empresas o negocios de las localidades donde se ubican las secciones del CRA que, no teniendo una relación directa como proveedores del CRA, sí que proveen de servicios, por ejemplo, de alojamiento y restauración, al profesorado itinerante y no residentes en dichas localidades.

- Entorno social, que es un grupo amplio y más difuso que incluye, principalmente, a los vecinos de esas localidades y las asociaciones locales.

Una vez definidos los stakeholders del colegio, establecimos un diálogo con cada grupo, a través de entrevistas en profundidad y encuestas, obteniendo información sobre qué valor les aporta este CRA, siguiendo el formato propuesto por San-José y Retolaza (2016: 163). En concreto, el guion de las entrevistas se sustentaba en estos aspectos clave: percepción del entrevistado sobre los principales aspectos en los que este CRA le generaba valor de forma individual o a su organización, según el caso; características que pueden hacer que el valor percibido se incremente; algún indicador concreto que pudiera utilizarse para identificar dicho valor percibido; y alguna cuantificación monetaria de referencia respecto a dicho indicador. En total se realizaron 16 entrevistas, en muchos casos telefónicas, con representantes de los grupos de interés. Número de entrevistas adecuado, dado el tamaño de la entidad analizada (menos de 50 trabajadores) y el nivel de heterogeneidad de los grupos de interés (medio-bajo). Según San-José y Retolaza (2016: 91-93), "en el límite inferior se debería hablar, al menos, con un interlocutor por grupo de interés. De nuestra experiencia se podría deducir que, para entidades de tamaño medio (50-250 trabajadores), entre quince y veinticinco entrevistas parece un número correcto; [...]. En la medida en que éstos sean más homogéneos, menor será el número de entrevistas a realizar" y viceversa. Además, la finalidad de estas entrevistas en profundidad es identificar las variables de valor y, en nuestro caso, se observó que las últimas entrevistas no aportaban variaciones en el grupo de variables de valor identificadas, habiendo llegado a lo que se conoce como saturación teórica (Strauss, 1987).

Adicionalmente, por su naturaleza de stakeholders principales, diseñamos tres encuestas, una para cada uno de los tres colectivos principales del colegio: alumnos, profesores y familias; que distribuimos a todos los miembros de estos colectivos, donde preguntábamos por algún indicador que pudiera utilizarse para identificar el valor generado, tipo "diferencia de edad entre grupos de amigos", "no de familias que se irían del pueblo si no hubiera CRA" o "el gasto medio por curso de los profesores en los pueblos del CRA", y por alguna cuantificación monetaria de referencia, como "el coste de una actividad extracurricular" o "la indemnización por km. de desplazamiento en coche propio por razones de servicio". Finalmente, se consiguió información de todos los profesores del CRA tanto del curso 2018-19 como del 2019-20, así como del equipo directivo (27 profesores), 12 niños/as de distintos niveles educativos y 21 padres/madres. 
A partir de dicha información, identificamos 13 variables de valor con sus correspondientes indicadores, que sintetizamos en la Tabla 4. A continuación describimos las variables, los indicadores y los proxis utilizados para su monetización:

- La educación básica, tiene que ver con la transferencia de valor que la enseñanza pública genera, valor que nada tiene que ver con su gratuidad. En este caso, usamos el número de estudiantes durante el período de referencia como indicador y, aunque el precio sombra podría ser un buen proxy, hemos utilizado la diferencia entre el gasto medio anual de las familias en un colegio privado y uno público, basándonos en el último informe de la OCU (2019). Siendo fieles a la filosofía que subyace a todo el modelo de San-José y Retolaza (2016), se ha optado por el principio de prudencia que emana de los principios contables más tradicionales.

- La cohesión grupal se refiere al buen ambiente que hay entre estudiantes, sin problemas de acoso entre compañeros, dado que en las aulas hay niños de varias edades y los alumnos de más edad ejercen en la práctica de hermanos mayores con los pequeños, jugando juntos dentro y fuera del centro escolar. Con una media de 5,58 años de diferencia de edad entre los alumnos y sus 4 mejores amigos, este CRA está en sintonía con lo que promueven programas anti-acoso efectivos implantados en distintos colegios de primaria, tal como el programa TEI o el Kiva (El País, 2019) en el que los alumnos mayores tutorizan a los pequeños. Como proxy parece razonable usar el coste de implantar este tipo de programas en horas de formación tanto para profesores como para alumnos.

- La cercanía y trato personalizado del profesor se deriva de la propia configuración docente de un CRA en el que un profesor tiene en el aula a alumnos de varios niveles educativos y debe adaptarse a las necesidades y demandas de cada uno de ellos de forma personalizada. Además, en este CRA, la ratio de estudiantes por profesor es de 4,06. De ahí que el indicador usado sea el número de estudiantes, tanto de infantil como de primaria, y el proxy sea el incremento del gasto medio anual para las familias por clases reducidas, suponiendo un máximo de alumnos por clase en todos los niveles de $25 \mathrm{ni}$ ños/niñas.

- El intercambio generacional se relaciona con la convivencia tanto en el ámbito escolar, a través de actividades para los niños organizadas por vecinos jubilados, como fuera de él, dado el reducido tamaño y la configuración de la pirámide poblacional de los pueblos donde se ubica este CRA, en donde por cada alumno hay 8,1 personas de más de 65 años de media (INE, 2020). Según Whitehouse et al. (2020), la intergeneraterapia mejora las habilidades sociales de los niños, proporcionándoles mayor perspectiva del ciclo de vida y reduciendo sus prejuicios hacia la vejez. Además, las personas mayores mejoran su salud, reduciendo la sintomatología depresiva y aumentando el efectivo intercambio de afecto. El indicador usado es el número de actividades realizadas y el proxy, el coste de dicho tipo de actividades extracurriculares.

- El arraigo poblacional tiene que ver con la capacidad del CRA de retener a la población autóctona e incluso de atraer a población foránea. Las AA.PP. destinan recursos tanto 
para prevenir el despoblamiento como para incentivar a las familias que deciden vivir en el medio rural. Así, distintas Comunidades Autónomas dan incentivos directos a las familias para reducir el problema de despoblación de muchas zonas rurales de España (Almanatura, 2019), como el cheque por cada hijo menor de edad o bonificaciones en el alquiler de la vivienda, para familias con hijos residentes en el medio rural. En nuestro caso, hemos detectado que el CRA es una fuente de arraigo de la población, que podría tener un efecto similar a este tipo de ayudas o incluso ser más eficaz, dados los comentarios de los padres a este respecto. En este sentido, 19 de los 71 niños y sus respectivas familias han venido de fuera; sin embargo y dado hemos preguntado expresamente ese dato a las familias, el indicador es el número de familias que se irían de los pueblos de influencia del CRA si este no estuviera, mientras que como proxy podríamos usar la valoración equivalente de alguno de los incentivos demográficos comentados y que, en caso de no existir el CRA, la Administración Pública correspondiente podría ofertar para atraer o retener a la población en el entorno rural. En este caso, guiándonos por el principio de prudencia, hemos optado por el cheque por niño, dado que es de menor importe (16.800 €) que la bonificación en el alquiler de vivienda, que supondría un ahorro de $107.640 €$.

- La dinamicidad económica inducida indirecta se deriva de la actividad económica indirecta generada por los profesores del CRA a los negocios de la zona, sobre todo, a bares, restaurantes y hostales, dado que sólo 3 profesores residen en alguno de los pueblos del CRA, viniendo el resto de otros pueblos o de Albacete capital. El indicador es el gasto que el conjunto de profesores realiza tanto en temas de restauración y hostelería durante el curso escolar (dato que nos proporcionaron los profesores), como en kilómetros en desplazamientos entre los pueblos del CRA para cumplir con sus tareas docentes y de gestión. Para este último caso, el proxy utilizado es la indemnización por kilometraje que la Junta de Comunidades de Castilla-La Mancha (JCCM) paga por este tipo de desplazamientos.

- La conciliación familiar se refiere a la posibilidad que da la existencia del CRA a las familias de compatibilizar mejor vida personal y laboral, en ahorro de tiempo. En este sentido, el indicador usado es el tiempo/km. que se tarda en ir desde cada pueblo hasta Alcaraz (lugar donde deberían acudir los niños si no existiera el CRA), según Google maps. El proxy es el salario mínimo interprofesional por hora. Para el cálculo se ha tenido en cuenta el número de familias que viven en cada pueblo y que estas deberían realizar dos viajes al día.

- La autonomía y tranquilidad tiene que ver con el buen clima laboral existente entre los profesores del CRA, que mejora su productividad e implicación con los alumnos. El indicador es el número de horas extras dedicadas a la tarea docente por parte de los profesores, por encima de la jornada laboral estipulada legalmente de 37,5 horas. El proxy es el salario medio bruto por hora de los profesores del CRA, según las órdenes 02/07/2012 05/08/2014 de la JCCM. 
- La promoción y movilidad laboral se refiere a las mayores opciones que se tiene en este tipo de colegios de conseguir puntos para los concursos públicos. Aunque en Castilla-La Mancha no se reconocen puntos extras por el hecho de dar docencia en un colegio de este tipo, en otras Comunidades Autónomas, como Aragón, sí que se dan más puntos por ejercer la profesión docente en pueblos pequeños (Heraldo, 2017). El indicador es el número de puntos por desempeño de cargos, según el Real Decreto 1364/2010, de 29 de octubre, y como proxy el número de horas de formación necesarias para conseguir el mismo número de puntos, sabiendo que se dan 0,1 puntos por cada diez horas de actividades de formación.

- El fomento de redes informales, tanto entre los alumnos como entre el resto de stakeholders implicados, se relaciona con el aumento de contactos sociales derivados de las interacciones propiciadas por el CRA. En este sentido, dos son los indicadores usados: para los alumnos, el porcentaje de alumnos cuyos amigos son del colegio; y para el resto, el número de visitas turísticas de los profesores del CRA en la comarca de influencia del CRA. Los proxis respectivos son el coste de gestionar redes sociales tipo Instagram, Facebook o Youtube (Cebolla, 2020) y los gastos de gestión y/o búsqueda de una agencia de viajes (Bulla, 2019).

- La calidad e innovación educativa tiene que ver con los programas educativos y los proyectos de innovación en los que participa este CRA. En concreto, participa en dos: Erasmus+ (SEPIE) y Tecno-CRA, cuyo principal resultado es el diseño, desarrollo e implementación de un software educativo gamificado que permite, entre otras funcionalidades, el seguimiento online del progreso de los alumnos y la integración y socialización de los mismos entre las distintas secciones del CRA. El proxy usado es la capacidad de captación de fondos que tiene, en términos de subvención recibida y de coste del software personalizado.

- La comunicación fluida con el entorno social se deriva de las buenas relaciones del personal del CRA con alcaldes y demás autoridades, asociaciones locales y vecinos en general. El indicador es el número de stakeholders que lo señalan como relevante, siguiendo a Román et al. (2020), y el proxy usado es el coste de gestionar redes sociales, tipo Instagram, Facebook o Youtube, pues estas son canales de comunicación ágil.

- Por último, la experiencia docente intensa se relaciona con el hecho de que un mismo profesor tiene en el aula niños de varios cursos al mismo tiempo, por lo que en pocos años estos profesores acumulan experiencia de diversas asignaturas y cursos similares a profesores con más años de ejercicio de la profesión. Así, el indicador es la media del número de cursos diferentes que se concentran en una misma aula y el proxy tiene que ver con los puntos que, por Resolución de 07/02/2019 JCCM, se obtienen por años de experiencia docente en distinto nivel o etapa educativa y el equivalente en horas de formación que deben realizar que se valoran en función del salario medio bruto por hora de los profesores del CRA. 


\begin{tabular}{|c|c|c|c|c|c|c|c|}
\hline $\begin{array}{l}\tilde{g} \\
\stackrel{\Xi}{ \pm} \\
\stackrel{\Xi}{\vec{U}}\end{array}$ & 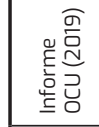 & 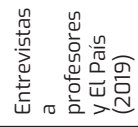 & 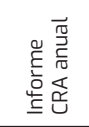 & 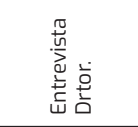 & 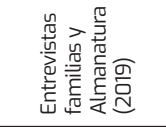 & 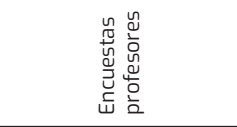 & 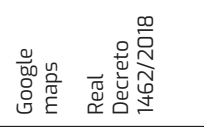 \\
\hline 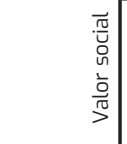 & 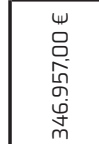 & 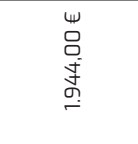 & 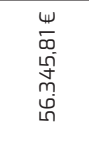 & 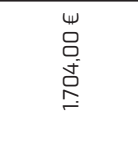 & 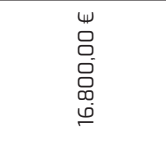 & 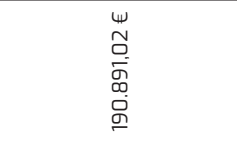 & 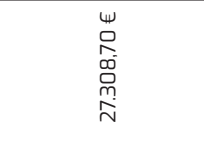 \\
\hline $\begin{array}{l}\vec{x} \\
0 \\
\vdots \\
0\end{array}$ & 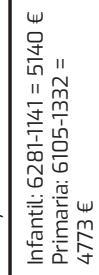 & 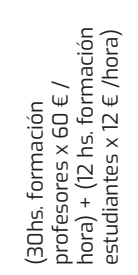 & 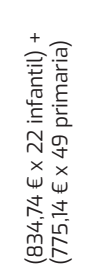 & 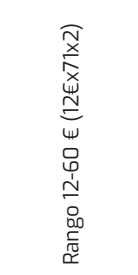 & 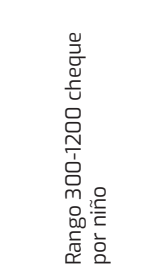 & 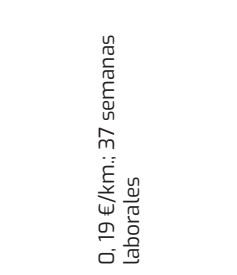 & 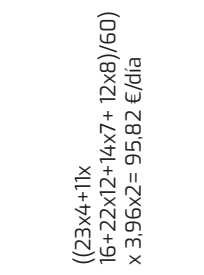 \\
\hline 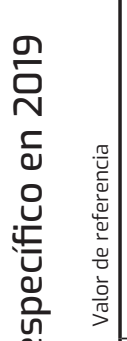 & 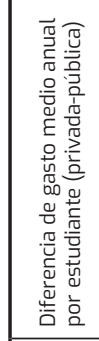 & 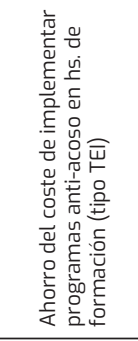 & 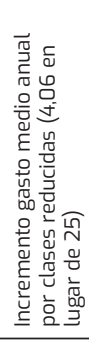 & 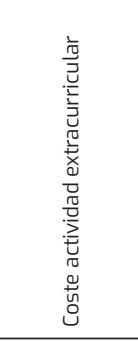 & 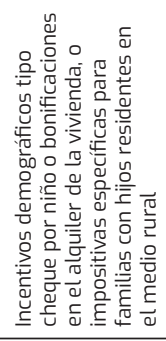 & 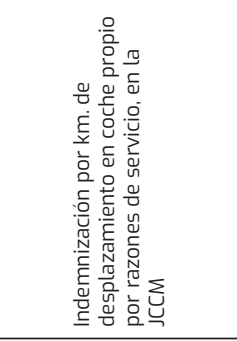 & 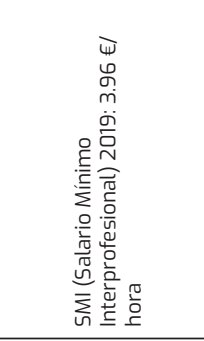 \\
\hline 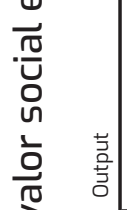 & 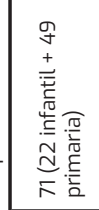 & 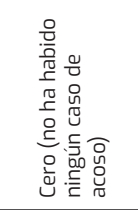 & 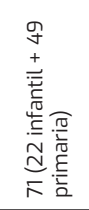 & $N$ & 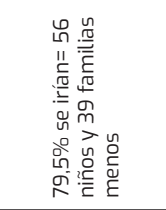 & 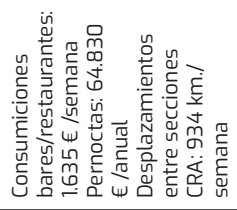 & 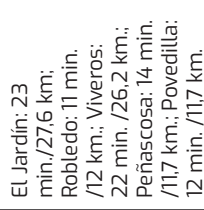 \\
\hline 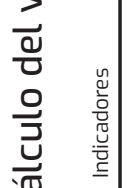 & 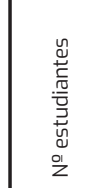 & 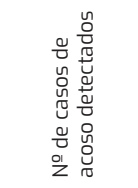 & 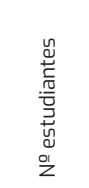 & 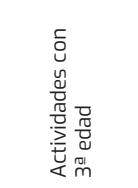 & 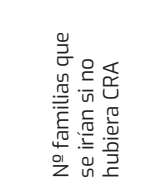 & 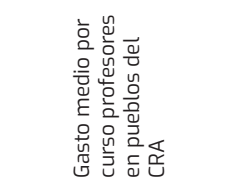 & 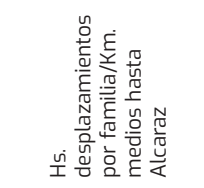 \\
\hline 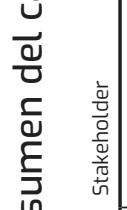 & 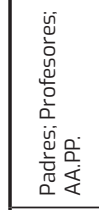 & 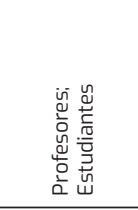 & 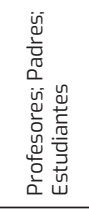 & 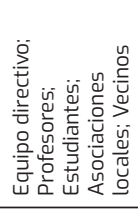 & 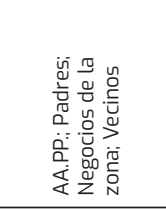 & 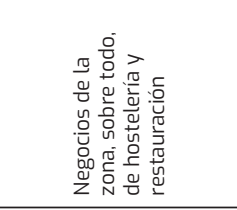 & $\begin{array}{l}\text { 凹 } \\
\frac{\square}{\pi} \\
\square\end{array}$ \\
\hline 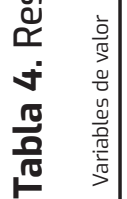 & 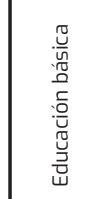 & 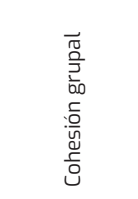 & 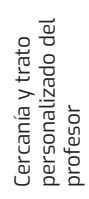 & 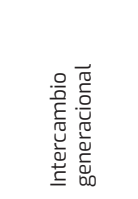 & 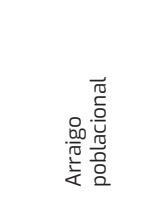 & 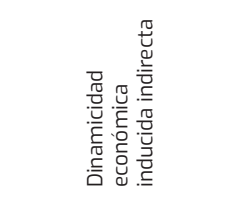 & 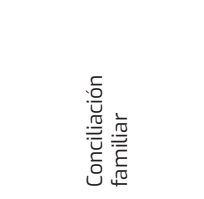 \\
\hline
\end{tabular}




\begin{tabular}{|c|c|c|c|c|c|}
\hline 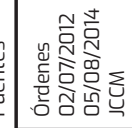 & 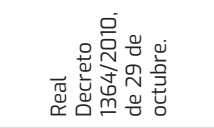 & 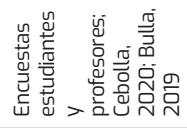 & 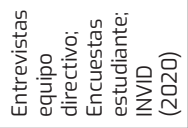 & 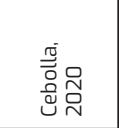 & 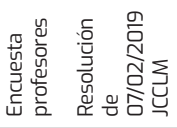 \\
\hline 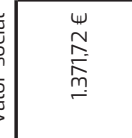 & 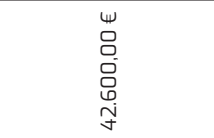 & 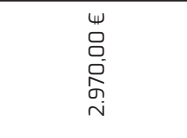 & 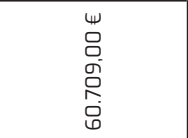 & 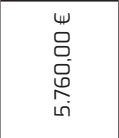 & $\begin{array}{l}\Psi \\
\text { O } \\
\text { ō } \\
\text { N̦ } \\
\stackrel{=}{=}\end{array}$ \\
\hline 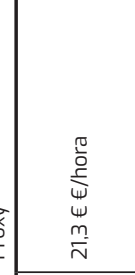 & 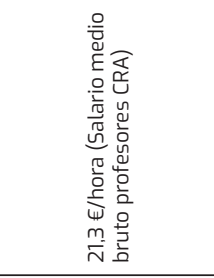 & 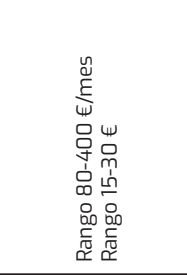 & 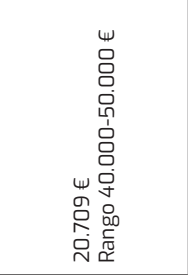 & 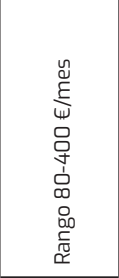 & 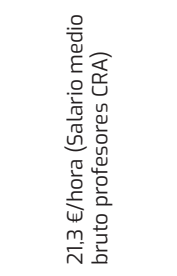 \\
\hline 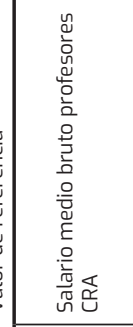 & 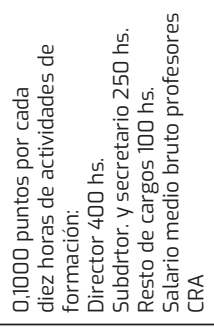 & 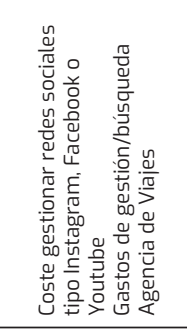 & 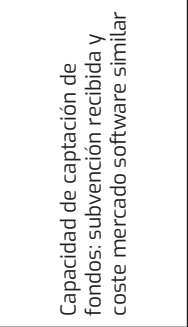 & 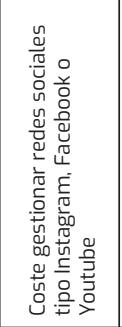 & 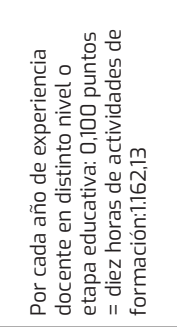 \\
\hline \begin{tabular}{l} 
ஸ் \\
ப் \\
\multirow{J}{*}{}
\end{tabular} & 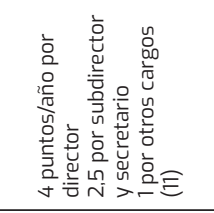 & 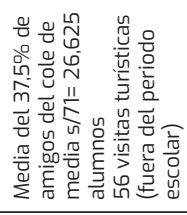 & 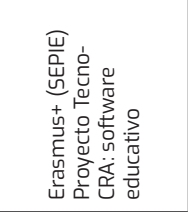 & & 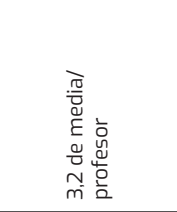 \\
\hline 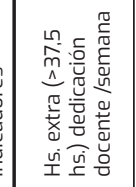 & 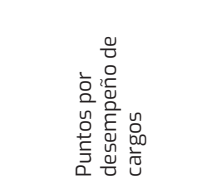 & 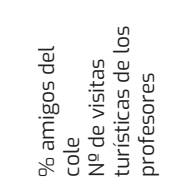 & 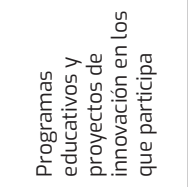 & 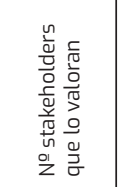 & 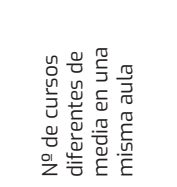 \\
\hline 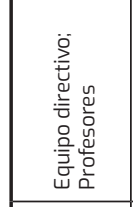 & 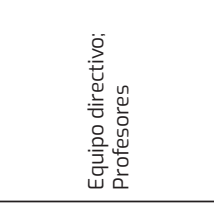 & 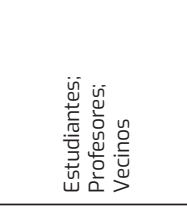 & 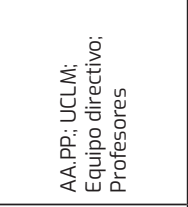 & 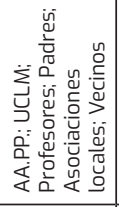 & 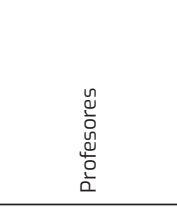 \\
\hline 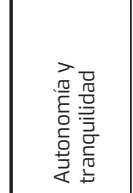 & 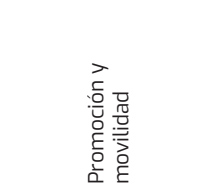 & 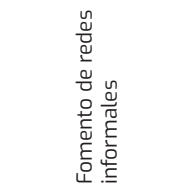 & 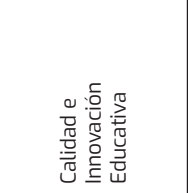 & 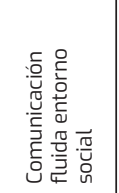 & 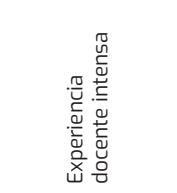 \\
\hline
\end{tabular}




\section{c. Valor Social Integrado}

Adicionando el Valor Social de Mercado (VSM) y el Valor Social Específico (VSE) obtenemos el Valor Social Integrado (VSI), en terminología de Retolaza, San-Jose y Ruiz-Roqueñi (2016). La Tabla 5 muestra el resumen de todos estos valores, siendo el VSI del CRA Sierra de Alcaraz de 1.624.487 € para el ejercicio económico de 2019.

\section{Tabla 5. Valor Social Integrado por el CRA Sierra de Alcaraz, 2019}

\begin{tabular}{l|r} 
Ecosistemas de Valor & Valor monetario \\
\hline Valor Social de Mercado & $857.148 €$ \\
\hline Valor Socio-Económico & $847.608 €$ \\
\hline Valor Movilizado & $9.540 €$ \\
\hline Valor Social Específico & $767.289 €$ \\
\hline Valor Social Integrado & $1.624 .437 €$ \\
\hline
\end{tabular}

Fuente: Elaboración propia.

Respecto a la distribución de dicho valor social integrado entre los principales stakeholders del CRA, las Administraciones Públicas reciben $276.343,70 €$, de los cuales $170.086 €$ son retorno directo vía impuestos diversos; las familias, aglutinando padres y alumnos, reciben un total de 455.087,81 €; y los trabajadores, tanto del CRA como de sus proveedores, 679.220,72 $€$. Además, los negocios de los pueblos de la zona de influencia del CRA obtienen rentas por valor de 190.891,02 €, gracias a las consumiciones, pernoctas y desplazamientos de los profesores. 
Tabla 6. Distribución del Valor Social Integrado del CRA Sierra de Alcaraz a sus stakeholders, 2019

\begin{tabular}{l|r|r|r} 
Stakeholders & \multicolumn{1}{r}{$\begin{array}{r}\text { Valor Social de } \\
\text { Mercado }\end{array}$} & $\begin{array}{r}\text { Valor Social } \\
\text { Específico }\end{array}$ & $\begin{array}{r}\text { Valor Social } \\
\text { Integrado }\end{array}$ \\
\hline Administraciones Públicas & $170.086,00 €$ & $106.257,70 €$ & $276.343,70 €$ \\
\hline Profesores + equipo directivo & $617.343,00 €$ & $58.829,72 €$ & \\
\hline Padres/familias & $46.559,00 €$ & $348.397,00 €$ & \\
\hline Alumnos & & $60.131,81 €$ & \\
\hline Negocios de la zona & & $190.891,02 €$ & \\
\hline Otros stakeholders & $23.160,00 €$ & $2.781,75 €$ & \\
\hline Total & $\mathbf{8 5 7 . 1 4 8 , 0 0 €}$ & $\mathbf{7 6 7 . 2 8 9 , 0 0 €}$ & $\mathbf{1 . 6 2 4 . 4 3 7 €}$ \\
\hline
\end{tabular}

Fuente: Elaboración propia.

\section{Conclusiones}

En este trabajo se ha cuantificado el valor social integrado del CRA Sierra de Alcaraz, ubicado en la provincia de Albacete, siguiendo la metodología del Modelo Poliédrico (SPOLY) de San-Jose y Retolaza (2016).

La monetización del valor social integrado en los CRA es un campo inexplorado en la aplicación de esta metodología y permite hacer visible, en términos monetarios, el valor íntegro atribuible a su gestión, no sólo la aportación económica que realizan, sino su contribución social. Ello podría conducir a un mayor reconocimiento de este tipo de entidades por parte del conjunto de la sociedad y de las Administraciones Públicas, lo que posibilitaría el diseño y la puesta en práctica de acciones específicas de mejora de los servicios de bienestar básicos, como la educación, en las zonas rurales, ayudando a evitar su despoblación. En este sentido, varios de los stakeholders del CRA (en conceto, Administración Públicas, Padres, Negocios de la zona y Vecinos) nos hicieron comentarios en el sentido de que "el CRA daba vida al pueblo" (por ejemplo, porque muchos profesores no residentes venían en periodo no escolar con amigos y familiares o porque muchos padres tenían claro que si no estuviera el CRA se mudarían de pueblo), ratificando lo apuntado por la literatura (Domínguez Álvarez, 2020; Escalona Orcao \& Díez Cornago, 2005; Boix, 2003).

Además, también valoraban muy positivamente prácticamente todos los stakeholders la buena relación entre todos ellos, es decir, la comunicación fluida entre todos ellos que fomentaba la creación de redes informales (de amistad), más allá de las puramente académicas, entre estudiantes de distintas edades, profesores, vecinos e incluso representantes instituciones, como los alcaldes Dicha integración y armonía intergeneracional contribuyen, en palabras de 
los profesores, a crear sentido de pertenencia en los alumnos, además de educarlos en valores, tales como el respeto a sus mayores y a no abusar de los más pequeños o de sus iguales. En este sentido, a pesar de que el acoso en las aulas es un problema cada vez más habitual en el contexto español (El País, 2019), en este CRA no se recuerda ningún caso. Intangibles que, como afirma Canals (2014), generan mayor valor que el derivado del servicio tangible que ofrecen de forma explícita.

A pesar de que se trata de una primera aproximación, el valor social específico del CRA Sierra de Alcaraz obtenido para el año 2019 (767.289 €) se aproxima al valor social de mercado derivado de su actividad principal en ese ejercicio (857.148€). Asimismo, cabe señalar que el valor social integrado de 1.624.437 € del CRA Sierra de Alcaraz representa casi el doble del importe recibido de financiación pública (828.067 €), lo que supone que por cada euro que las AA.PP. invierten en este centro, el CRA Sierra de Alcaraz genera, al menos, $1.96 €$. Teniendo en cuenta que en el cálculo del valor social específico se ha optado siempre, siguiendo el principio de prudencia, por el menor valor del rango de los valores razonables para cada proxy, la aproximación al VSI ha sido por defecto.

Respecto a las limitaciones de este trabajo de investigación, la primera se deriva de la condición de institución docente pública que conlleva la inexistencia de una contabilidad unificada y homogénea, que ha supuesto, además, un verdadero reto para obtener las diversas partidas de la cuenta de pérdidas y ganancias necesarias para este estudio, al estar implicadas distintas AA.PP. Sin duda, la existencia de información pública, estandarizada y desagregada de los presupuestos completos de los centros docentes ayudaría, no sólo a la transparencia, sino también a la concienciación ciudadana del valor de los servicios públicos, aunque les resulten gratuitos. En segundo lugar, este trabajo se sustenta en el estudio de un solo caso, por lo que en futuras investigaciones debería ampliarse el número de casos analizados antes de poder concluir cuáles de las variables de valor aquí señaladas son comunes a todos los CRA y cuáles se derivan del contexto concreto del CRA Sierra de Alcaraz. Por último, la utilización de proxis supone asociar un valor razonable a una variable de valor no directamente medible en términos económicos, por lo que se asume la existencia de un gap o desviación con el valor real. Investigaciones futuras deben ir depurando estos proxis para reducir dicho gap. Por último, el valor social integrado no incluye el valor emocional, tan importante para la monetización del impacto social global generado por cualquier organización, sobre todo para aquellas de no mercado (Román Cervantes C. , Guzmán Pérez, Mendoza Jiménez, \& Pérez Monteverde, 2019). En futuras investigaciones sería conveniente la integración de variables de valor como la satisfacción profesional o el bienestar personal, muy repetidas entre algunos de los grupos de interés de este CRA.

En suma, el proceso SPOLY de Contabilidad Social permite comprender, medir y comunicar el valor creado por los CRA y, por tanto, es una herramienta útil para la planificación estratégica de este tipo de entidades y su comunicación con los distintos grupos de interés. Cabe destacar que el marco de indicadores y proxis utilizado en este estudio puede adaptarse a otros CRA. Por consiguiente, los resultados de este trabajo deben servir de estímulo para que más CRA integren en su gestión el análisis del valor social. Ello permitiría un mayor y mejor 
conocimiento del impacto social generado por estas entidades y una mejor comunicación de sus resultados a las AA.PP., de las que depende la mayor parte de su presupuesto. En este sentido, los responsables de las políticas educativas y de gestión del territorio deberían considerar el resultado obtenido por los CRA, en términos de rentabilidad y no sólo de coste, para decidir sobre su creación, mantenimiento o cierre en una determinada comarca.

\section{Bibliografía}

AGLE, B.R., DONALDSON, T., FREEMAN, R.E., JENSEN, M.C., MITCHELL, R.K. \& WOOD, D.J. (2008): "Dialogue: Toward superior stakeholder theory", Business Ethics Quarterly, 18(2), 153190.

ALMANATURA (2019): Coste ambiental, social y económico de la despoblación. Retrieved julio 14,2020 , from https://almanatura.com/2019/02/coste-ambiental-social-economico-despoblacion.

AYUSO, S., SÁNCHEZ, P., RETOLAZA, J.L. \& FIGUERAS-MAZ, M. (2020): "Social value analysis: the case of Pompeu Fabra University", Sustainability Accounting, Management and Policy Journal, 11(1), 233-252. D0I:10.1108/SAMPJ-11-2018-0307.

BOIX, R. (2003): "Escuela rural y territorio: entre la desruralización y la cultura local", Revista Digital eRural, Educación, cultura y desarrollo rural, Año 1(1).

BULLA, E. (2019): Gastos de gestión de las agencias de viaje. Retrieved julio 20, 2020, from https://www.preferente.com/noticias-de-agencias-de-viajes/gastos-de-gestion-las-agenciasno-saben-que-cobrar-287317.html

CANALS, C. (2014): Intangibles: la nueva inversión en la era del conocimiento. Informe Mensual-La Caixa, Departamento de Macroeconomía, Área de Planificación estratégica y Estudios, CaixaBank. Barcelona, España: CaixaBank Research.

CEBOLLA, F. (2020): Llevar redes sociales. Retrieved julio 25, 2020, from https://fernandocebolla.com/llevar-redes-sociales

COLLANTES, F., PINILLA, V., SÁEZ, L.A. \& SILVESTRE, J. (2010): El impacto demográfico de la inmigración en la España rural despoblada. Documento de trabajo , Real Instituto Elcano, Área Demografía y Población.

CONSEJO ECONÓMICO Y SOCIAL (2018): El medio rural y su vertebración social y territorial. Informe CES 01/2018, Madrid, España. 
CONSEJO ESCOLAR DEL ESTADO (2016): Informe 2016 sobre el estado y situación del sistema educativo. Curso 2014-2015, Ministerio de Educación, Cultura y Deporte, Madrid, España.

CONSEJO ESCOLAR DEL ESTADO (2019): Informe 2019 sobre el estado y situación del sistema educativo. Curso 2017-2018, Ministerio de Educación y Formación Profesional, Madrid, España.

DOMINGO CEBRIÁN, V. (2013): Origen y evolución del modelo CRA -Colegio Rural AgrupadoSituación en Aragón y estudio de caso de la realidad turolense, Facultad de Filosofía y Ciencias de la Educación, Departamento de Teoría de la Educación, Valencia: Universitat de València.

DOMÍNGUEZ ÁLVAREZ, J.L. (2020): “El desigual acceso de la juventud rural a los servicios públicos: la necesidad de impulsar la educación en la España vaciada”, Cuadernos de Investigación en Juventud, 8, 60-78. DOI:10.22400/cij.8.e047.

EL PAÍS (2019): Programas antiacoso en el colegio. Retrieved july 8, 2020, from https://elpais.com/elpais/2019/03/07/mamas_papas/1551948149_631118.html.

ESCALONA ORCAO, A.I. \& DÍEZ CORNAGO, C. (2005): "Retos y problemas de la accesibilidad a servicios en zonas despobladas: un caso en la provincia de Teruel (España)", Scripta Nova: revista electrónica de geografía y ciencias sociales, 9(2).

ESCRIBANO PIZARRO, J. (2012): "Servicios educativos y sanitarios elementales en el medio rural: percepción social e influencia sobre la calidad de vida", Revista de Estudios Geográficos, 73(272), 35-61. DOI:10.3989/estgeogr.201202.

ETXEZARRETA, E., MENDIGUREN, J.C., DÍAZ, L. \& ERRASTI, A. (2018): “Valor social de las cooperativas sociales: aplicación del modelo poliédrico en la cooperativa para la acogida de menores Zabalduz S.Coop.", CIRIEC-España, Revista de Economía Pública, Social y Cooperativa, 93, 155-180, DOI: 10.7203/CIRIEC-E.93.9953.

FEU I GELIS, J. (2004): "La escuela rural en España: apuntes sobre las potencialidades pedagógicas, relacionales y humanas de la misma", Revista Digital eRural, Educación, cultura y desarrollo rural, 2(3), 1-13.

FREEMAN, E. (1984): Strategic management a stakeholder approach, Boston, United States: Pitman.

FREEMAN, E., RETOLAZA, J.L. \& SAN-JOSÉ, L. (2020): "Stakeholder Accounting: hacia un modelo ampliado de contabilidad", CIRIEC-España, Revista de Economía Pública, Social y Cooperati$v a, 100,89-114$. DOI: $10.7203 /$ CIRIEC-E.100.18962.

FREEMAN, E., WICKS, A.C., \& PARMAR, B. (2004): "Stakeholder Theory and The Corporate Objective Revisited", Organization Science, 15(3), 364-369, D0I: 10.1287/orsc.1040.0066. 
GUZMÁN PÉREZ, B., MENDOZA JIMÉNEZ, J., PÉREZ MONTEVERDE, M.V. \& ROMÁN CERVANTES, C.A. (2018): "El valor social de las cofradías de pescadores de Canarias", Revista de la Asociación Española de Contabilidad y Administración de Empresas, 124, 18-21.

HERALDO (2017): Los profesores que vayan a pueblos más pequeños y más alejados tendrán más puntos. Retrieved july 21, 2020, from

https://www.heraldo.es/noticias/aragon/2017/10/30/los-profesores-que-vayan-pueblos-mas-pequenos-alejados-tendran-mas-puntos-1204300-300.html

INE (2020): Cifras oficiales de población de los municipios españoles: Revisión del Padrón Municipal. Instituto Nacional de Estadística, Madrid, España.

INVID (2020): Cuánto cuesta el software personalizado. Retrieved july 15, 2020, from https://invidgroup.com/es/cuanto-cuesta-el-software-personalizado/\#: :text=Normalmente $\% 2 \mathrm{C} \% 20 \mathrm{el} \% 20$ costo $\% 20 \mathrm{del} \% 20$ desarrollo,del $\% 20$ desarrollo\%20de $\% 20$ software $\% 20$ personalizado,

JESUS, D.S. (2020): "Three Tales of Capitalism and Creative Economy", International Relations, $8(2), 39-44$.

LACORT NAVARRO, C. (2014): La identidad del alumnado en la práctica docente diaria actual en un Colegio Rural Agrupado. Trabajo fin de Grado, Universidad Internacional de La Rioja, Facultad de Educación, Huesca, España.

LEWIN, K. (1946): "Action research and minority problems", Journal of social issues, 2(4), 3446.

MINISTERIO DE EDUCACIÓN Y CIENCIA (1986): Real Decreto 2731/1986, de 24 de diciembre, sobre Constitución de Colegios Rurales Agrupados, Madrid, España: BOE no 8 de 9 de enero de 1987.

OCU (2019): Gastos escolares del nuevo curso. Retrieved junio 23, 2020, from https://www.ocu.org/consumo-familia/bebes/informe/gastos-escolares-nuevo-curso

PONCE DE LEÓN ELIZONDO, A., BRAVO SÁINZ, E. \& TORROBA SANTA MARÍA, T. (2000): “Los Colegios Rurales Agrupados, primer paso al mundo docente", Contextos Educativos, 3, 315-347.

PORTER, M. \& KRAMER, M.R. (2011): Creating shared value, Harvard Business Review, 4, 1-17.

RETOLAZA, J.L., AGUADO, R. \& SAN-JOSE, L. (2020): "Social Accounting as an Enabling Tool to Develop Collective Organizational Citizenship Behavior in the Diocese of Bilbao", Frontiers in Psychology, 11, 77, 1-11 D0I: 10.3389/fpsyg.2020.00077. 
RETOLAZA, J.L., RUIZ-ROQUEÑI, M., SAN-JOSÉ, L. \& BARRUTIA, J. (2014): “Cuantificación del valor social: propuesta metodológica y aplicación al caso de Lantegi Batuak", Zerbitzuan. Revista de servicios sociales, 55, 17-33, DOI: 10.5569/1134-7147.55.02.

RETOLAZA, J.L., SÁNCHEZ, P., TARIFA, P., RUIZ-ROQUEÑI, M., SAN-JOSÉ, L. \& AYUSO, S. (2015): Análisis del valor social de la Fundación Formació $i$ Trebal: Una aproximación monetaria al cálculo del valor social. Working Paper Cátedra Mango, 22. Barcelona, España.

RETOLAZA, J.L., SAN-JOSÉ, L. \& RUIZ-ROQUEÑI, M. (2015): “Monetarizing the social value: Theory and evidence", CIRIEC-España, Revista de Economía Pública, Social y Cooperativa, 83, 43-62.

RETOLAZA, J.L., SAN-JOSÉ, L. \& RUIZ-ROQUEÑI, M. (2016): Social Accounting for Sustainability: Monetizing the Social Value. Heidelberg, Germany: Springer.

ROMÁN CERVANTES, C.A., GUZMÁN PÉREZ, B., MENDOZA JIMÉNEZ, J. \& PÉREZ MONTEVERDE, M.V. (2020): "La sostenibilidad social de las SATs: una propuesta de indicadores para su evaluación", REVESCO, Revista de Estudios Cooperativos, 133, 71-80, D0I: 10.5209/reve.67336.

ROMÁN CERVANTES, C., GUZMÁN PÉREZ, B., MENDOZA JIMÉNEZ, J. \& PÉREZ MONTEVERDE, M.V. (2019): Informe del valor social de la SAT FAST-2017, Cátedra Cajasiete de Economía Social y Cooperativa, Universidad de La Laguna, Santa Cruz de Tenerife, España.

RUIZ-ROQUEÑI, M. (2018): Valor emocional. Medición del Valor Social para la Sostenibilidad, Curso de Verano de la Universidad del País Vasco, 5-6 de julio, Universidad del País Vasco.

SAN-JOSE, L. \& RETOLAZA, J.L. (2016): Contabilidad Social orientada a los stakeholders. Perspectiva de la Administración Pública. Madrid, España: Ed. Pirámide.

SAN-JOSE, L., RETOLAZA, J.L. \& BERNAL, R. (2019): "Índice de valor social añadido: una propuesta para analizar la eficiencia hospitalaria", Gaceta Sanitaria, 1-7.

DOI: 10.1016/j.gaceta.2019.08.011.

SAN-JOSE, L., RETOLAZA, J.L. \& FREEMAN, R.E. (2017): “Stakeholder Engagement at Extanobe: A Case Study of the New Story of Business". In: R. Freeman, J. Kujala, \& S. Sachs, Stakeholder Engagement: Clinical Research Cases (pp. 285-310). Issues in Business Ethics, vol 46: Springer, Cham. D0I:10.1007/978-3-319-62785-4_13.

STRAUSS, A. (1987): Qualitative Analysis for Social Scientists. Cambridge, Reino Unido: University of Cambridge Press.

SSPA (2018): Documento de posición de la Red de Áreas Escasamente Pobladas del Sur de Europa (SSPA) para España, Southern Sparsely Populated Areas (SSPA), Cuenca, España.

VIDAL MARTÍNEZ, A. (2014): “El precio de lo gratuito", Informe Mensual-La Caixa no 384, Barcelona, España. 